\title{
¿Qué historia para qué ciudadanía? La enseñanza de la historia en la educación básica en México
}

\author{
Which history, to which citizens? History teaching in \\ basic education in Mexico
}

\section{Que história, para qual cidadania? O ensino de história na educação básica no México}

\author{
Xavier Rodríguez Ledesma*
}

\begin{abstract}
Resumen: En México la reflexión acerca de los vínculos entre la historia como disciplina escolar y los procesos sociales de construcción de ciudadanía, requiere concebir este objeto de estudio como sólo una parte de un proceso mucho más complejo que lo vincula tanto a la educación cívica y ética, como a las prácticas cotidianas conformadoras de una cierta cultura escolar. Es necesario ubicar históricamente los orígenes y formas de funcionamiento de cada una de esas facetas, así como la manera en que entre ellas se refuerzan en sus afanes formativos. Sólo de esa forma es posible reconocer los fines, utilidades y límites de la enseñanza de la historia tal cual es concebida hoy en día, así como su imposibilidad de asumir como objetivos educativos la construcción de nociones democráticas referidas a una concepción moderna de ciudadanía.
\end{abstract}

Palabras claves: Ciudadanía. Historia y su enseñanza. Formación para la democracia.

\begin{abstract}
In Mexico, a reflection on the links between History as a school subject and the processes of social construction of citizenship requires conceiving the object of study as only part of a much more complex process that binds it both to civic education and ethics, and to everyday forming practices of a certain school culture. It is necessary to historically locate the sources and forms of operation of each of these facets as well as the way they reinforce their training claims. Only in this way it is possible to recognize purposes, uses and limits of teaching History as it is conceived today, as well as its inability to take as an educational goalthe building of democratic notions related to a modern conception of citizenship.
\end{abstract}

Keywords: Citizenship. History and its teaching. Training for democracy.

Resumo: No México a reflexão acerca dos vínculos entre a história como disciplina escolar e os processos sociais de construção de cidadania, requer conceber este objeto de estudo como apenas uma parte de um processo muito mais complexo que o

\footnotetext{
Licenciado y maestro en sociología. Doctor en ciencia política. Miembro del Sistema Nacional de Investigadores. Co-coordinador del Seminario Permanente de Investigación sobe la Enseñanza de la Historia y las Ciencias Sociales (SPIEHCS). E-mail: <xavirole@gmail.com>
} 
vincula tanto à educação cívica e ética, como às práticas cotidianas conformadoras de uma certa cultura escolar. É necessário situar historicamente as origens e formas de funcionamento de cada uma dessas facetas, assim como a maneira em que entre elas se reforçam em suas pretensões formativas. Somente desse modo é possível reconhecer os fins, utilidades e limites do ensino de história tal qual é concebido hoje em dia, assim como sua impossibilidade de assumir como objetivos educativos a construção de noções democráticas referidas a uma concepção moderna de cidadania.

Palavras-chave: Cidadania. História e seu ensino. Formação para a democracia.

\section{Presentación}

Fue en los años ochenta del siglo pasado cuando las preguntas sobre la vigencia o no de las tradicionales funciones de la enseñanza de la historia empezaron a permear la reflexión especializada. Hasta entonces parecía haber consenso acerca de la legitimidad, validez y necesidad de que la enseñanza de la historia estuviera vinculada directamente a la creación de las identidades nacionales. Así había sido desde su surgimiento como disciplina escolar en el siglo XIX. Sin embargo, el mundo súbitamente se trastocó, la historia pareció encontrar nuevos derroteros por donde la humanidad habría de andar y con ello provocó que ella misma vista como disciplina escolar tuviera que empezar a poner en el centro de la discusión la necesidad de resignificar su utilidad para las sociedades contemporáneas.

Entre los fenómenos históricos que en las últimas tres décadas hemos atestiguado podemos contemplar los siguientes hechos:

a) La caída del muro de Berlín y el fin del mundo socialista,

b) el incremento de las grandes corrientes migratorias de habitantes venidos de países de Europa oriental, de Latinoamérica y de África -tanto de los países mediterráneos como de las excolonias subsaharianas- que se dirigen a Europa y/o Estados Unidos en busca de trabajo y mejor nivel de vida

c) la aparición de nuevas realidades y problemas por resolver a partir del inicio de los procesos de democratización de las sociedades con regímenes políticos autoritarios en Latinoamérica.

d) la insurrección indígena encabezada por el Ejército Zapatista de Liberación Nacional (EZLN) en estado de Chiapas, México, en 1994.

e) la moderna globalización del mundo que trajo como una de sus consecuencias la instauración del mercado y formas culturales generadoras de nuevas identidades supranacionales.

f) La irrupción y retorno de nacionalismos particulares que habían sido subsumidos dentro de las nociones generales de Estado Nación o bloques geopolíticos. 
g) El surgimiento y/o reaparición de nuevas formas identitarias producto de todos los anteriores fenómenos sociales.

Todos esto obligó a poner en el centro de la reflexión social a temas que hasta ese momento, en el mejor de los casos, ocupaban roles secundarios en la reflexión social, por ejemplo: a) el reconocimiento de la necesidad de pensar y vincularse de maneras distintas con la otredad (PAZ, 1993; RODRÍGUEZ, 2009), b) el significado del surgimiento de nuevas identidades (MAALOUF, 2008), c) la comprensión y reconocimiento de que la historia es tan sólo una construcción, una narración, cuyo sentido de objetividad tiene que ver no con cuestiones de cientificidad sino con razones de poder (WHITE, 1992a, 1992b; DE CERTEAU, 1993, 1995; FERRO, 2000), d) la identificación de la historia tan sólo como una de las diversas facetas a través de las cuales el poder construye su hegemonía cultural (SAID, 2004a, 2004b; BERNAL, 1993), e) la necesidad de construir y difundir las otras historias que existen pero no forman parte de la historia hegemónica (TENORIO 2009; PLÁ, 2009; FLORESCANO, 2012; CARRETERO, 2007) la apertura a la posibilidad de pensar un currículo escolar que abrogue la división disciplinarias reformulando así todo el sentido del proceso de aprendizaje y la institución escolar misma (CONAFE, 1999; CUESTA, 2003), etcétera.

Junto con lo anterior, las nuevas formas de asumir la coexistencia entre los distintos -entre todos los otros- ubicaron como puntos fundamentales e ineludibles a problemáticas inéditas referidas a las formas de convivencia (tolerancia, educación para la paz, resolución de conflictos, etc.), lo cual necesariamente llevó a repensar cuál habría de ser el significado contemporáneo (características, obligaciones, derechos, necesidades, objetivos) de ser ciudadano en estas sociedades complejas, diversas, con anhelos de democracia, de tintes mundiales, etcétera.

Bajo este amplio y complejo contexto histórico, social y cultural, la historia en particular y las ciencias sociales en general como disciplinas escolares empezaron a ser cuestionadas. La razón era evidente ¿ellas (la historia, la geografía, la educación cívica,) debían seguir manteniendo sus añejos propósitos disciplinarios o habrían de sufrir cambios tan radicales como los que la sociedad ha vivido? Es decir, ¿la función social de la historia, su utilidad, ha de continuar siendo referida y atada a la construcción de identidades nacionales de características decimonónicas, o ella debiera repensarse a fin de adoptar una forma totalmente diferente que coadyuve a la resolución de los conflictos de convivencia que las nuevas condiciones sociales han y continúan generando?

Los estudios acerca de los procesos de enseñanza aprendizaje de la historia, paulatinamente y no sin grandes resistencias, han integrado a sus preocupaciones 
las reflexiones sobre las nuevas competencias que el conocimiento histórico escolar ha de generar, construir y promover, vinculándolas a la construcción de una educación ciudadana entendida ésta como un proceso de formación política y moral que desarrolle:

[...] actitudes, valores, principios y normas de convivencia democrática, así como de competencias para la participación responsable en la construcción de la sociedad. [...] implica formar niñas, niños y jóvenes con valores cívicos, capacidad de indignación ante las injusticias y abusos, sentido de responsabilidad ciudadana y capaces de convivir en la diversidad. (CONDE, 2009, p. 67-68).

Actualmente este tipo de preocupaciones permean el horizonte reflexivo. Las especificidades de las problemáticas socioculturales de las sociedades desde las cuales se discute qué historia debe enseñarse hoy día, hace que en los distintos países la discusión adopte características particulares. Así, generalizando, en Estados Unidos las ideas giran alrededor de la necesidad de contar las otras historias, particulares y específicas de los grupos excluidos de la historia oficial. En España, además de la recuperación de las historias particulares de las diversas regiones territoriales se pueden encontrar interesantísimos esfuerzos por romper los nichos disciplinarios y avanzar en la construcción de un conocimiento social que ayude a construir normas de convivencia plurales y democráticas para salir al paso de los problemas que la interculturalidad y diversidad social generan. Lo mismo puede decirse de Latinoamérica donde, además, la historia de los excluidos (indios, negros, mujeres, jóvenes, asalariados, etcétera) exige cada vez más su aparición en las narraciones escolares.

A continuación desarrollaré con mayor detalle la forma en que el tema de la historia y la educación ciudadana se presenta hoy en día en México. Obvio es que muchas de las características aquí referidas no son exclusivas a esta sociedad, al contrario, son expresiones autóctonas de problemas compartidos a nivel mundial, según acabamos de ver.

En el presente trabajo partimos de una idea básica: el análisis de qué tipo de historia es la que tradicionalmente se ha enseñado, sus utilidades y funciones sociales, así como sus vínculos con la construcción de un cierto tipo de ciudadano, va más allá de la reflexión sobre los contenidos específicos de los programas disciplinares, abarcando un universo bastante más amplio. En México, la respuesta a la pregunta sobre los vínculos entre la historia que se enseña en la escuela y los procesos de construcción de ciudadanía, necesariamente implica ver la forma en que esa disciplina es tan sólo una parte de un proceso mucho más complejo en donde, junto a la educación cívica y ética y a las prácticas cotidianas generadoras de una cierta cultura escolar, conforman los tres ejes definidores 
del proceso. Hacer la historización de esa tríada permite identificar los fines, utilidades y límites de la enseñanza de la historia, así como la imposibilidad de que ella, tal cual es concebida y tratada como disciplina escolar, asuma como objetivo la construcción de nociones democráticas referidas a una concepción moderna de ciudadanía.

De tal forma, es necesario poner bajo el lente del análisis crítico a toda la institución escolar, la cual se caracteriza justamente por la reproducción de prácticas políticas y sociales ajenas a un sentido democrático de convivencia. Así pues, si bien la reflexión sobre los problemas para construir ciudadanía pasa por analizar el tipo de historia y la forma en que es enseñada dentro de la escuela, rebasa por mucho esos estrechos límites, asignando tan grande responsabilidad a toda la institución escolar. Paulo Freyre (1971) décadas atrás lo tenía perfectamente claro y a partir de ahí muchos otros han bordado alrededor de este punto.

¿Se puede hacer algo desde las clases de historia para fomentar valores democráticos? ¿A la formación de qué tipo de ciudadanía abona la historia que se enseña hoy en día en el sistema escolar mexicano? ¿Esa historia puede ser "ajustada" para avanzar hacia la construcción de nociones democráticas modernas en los estudiantes? ¿Simplemente se requiere rediseñar los contenidos temáticos de la disciplina? Estas son algunas de las preguntas que pueden servir de punto de arranque a la reflexión.

Identifico tres rubros para el acercarnos al tema: a) la escuela y la formación de ciudadanía, b) los docentes y c) la concepción de historia.

\section{La escuela y la formación de ciudadanía}

Hipótesis a manera de provocación: mientras en la sociedad exista la discriminación en sus diversas expresiones, desde las más violentas físicamente hasta aquellas aparentemente inocuas y banales como la utilización de ciertos conceptos específicos como insultos (indio, naco, vieja, joto, prieto, marica, negro, sudaca, etc.) o, incluso, mientras se siga considerando de "buena educación" que una persona conteste con la frase "mande usted" "cuando es requerida por otra, seguirá evidenciándose el fracaso de las políticas educativas referidas al ámbito de la enseñanza aprendizaje de la historia y de la formación cívica y ética como las áreas que han de construir las competencias necesarias para la convivencia ciudadana moderna.

\footnotetext{
${ }^{1}$ En México la expresión "mande" o "mande usted” son frases consideradas de cortesía y buena crianza cuando alguien es interpelado. El sentido servil de dicha respuesta se ha rastreado hasta la época de la colonia cuando los indios convertidos en servidumbre debían contestar de esa manera a sus amos. El amo mandaba, el indio obedecía, luego entonces, éste siempre debía dirigirse a aquel preguntándole qué era lo que mandaba.
} 
El sistema educativo, la escuela, no puede ser señalada como la única responsable de ese fracaso social expresado en la instauración y reproducción de formas discriminatorias, intolerantes y violentas de convivencia. Es más, radicalizando la posición, ni siquiera ella es el factor de mayor peso en la explicación de la inexistencia en la población de las competencias necesarias para la convivencia pacífica y democrática. La escuela actual no está diseñada para eso, al contrario.

Una escuela para la democracia pasaría por la reformulación absoluta y radical de todo el sistema educativo, lo cual implica la realización de cambios sociales profundos que permitieran asignarle al sistema educativo funciones hegemónicas distintas a las que actualmente posee. Frente a esa idealidad hoy en día tenemos otro tipo de institución. A la escuela diseñada y construida para la homogenización y la normalización, para la generación de sujetos pasivos obedientes a la autoridad y las instituciones, no se le puede exigir que eduque para la tolerancia, el respeto a la diversidad, la participación activa de sus estudiantes en la toma de decisiones sobre su devenir, y demás responsabilidades que una noción moderna de ciudadanía implica. A una institución hecha para ser reproductora de una hegemonía específica y particular, es por demás conflictivo e incluso contra natura (ergo imposible) exigirle la generación de sentidos contra hegemónicos. Es sumamente complicado que en edificios hechos a modo de prisión se promueva una educación para la libertad. ¿Las mallas, rejas, los guardias ("prefectos" se les llama) etc., pretenden impedir que el afuera entre al espacio escolar, o que lo de adentro pueda escapar? La unanimidad abrumadora del monocolor ya sea gris o verde pistache, únicos tonos con los que está pintada la enorme mayoría de los edificios escolares del sistema de educación básica en México, elimina la pluralidad hasta en los colores usados en las paredes.

El uso del uniforme es obligatorio para los alumnos y alumnas de escuelas que a su vez están uniformadas incluso arquitectónicamente. Los intentos de diferenciación entre los sujetos no simplemente se desalientan sino que están prohibidos. A pesar de que la norma no lo plantea, en los hechos existe una serie de límites impuestos por la autoridad. Este punto no es menor, al contrario, se refiere a la naturalización de que la autoridad -encarnada en sus funcionariospueda imponer su juicio y criterio por encima de lo estipulado en las normas y las leyes. Es una faceta más de la instauración hegemónica de la noción de que el principio de autoridad está por encima de los derechos de los individuos.

La lucha cotidiana por la eliminación de la diferencia se expresa en multitud de prácticas: imposición de límites al tamaño del corte de pelo de los hombres o a la altura de las faldas de las mujeres, prohibición del uso de tatuajes, 
piercings, ciertos bailes, etc. Todos los días los alumnos deben formarse por grupos, por género y por estatura para dirigirse "ordenadamente" a los salones donde deberán guardar silencio mientras ocupan sus pupitres para atender con respeto al profesor o profesora que les dictará la clase, etc.

Todo ello constituye la imposición de un orden que anhela eliminar a la espontaneidad y a lo diferente. Se trata de homogeneizar, uniformar, de consolidar una concepción específica en la cual los roles de autoridad y ciudadanía están perfectamente definidos, donde los mecanismos de poder al interior reproducen las formas en que él se ejerce en el exterior. Es una institución diseñada para combatir lo distinto, lo extraño, lo ajeno, lo otro. Una escuela construida para fomentar la obediencia frente al poder y la autoridad no puede formar para la libertad la cual, al contrario, es vista como un factor negativo, peligroso, atentatorio de la continuidad de los sistemas de control y ejercicio del poder. Se trata entonces de no olvidar que la escuela es una de las instituciones conformadoras y reproductoras de la hegemonía, y dentro de ella la enseñanza de la historia ocupa un rol importante.

A todo lo anterior es necesario agregar que en México, como en muchos otros países, la construcción de un sistema educativo de índole nacional en el siglo XIX tenía como objetivo generar un sentido de Nación al consolidar la presencia de ese nuevo estado a través de llevar el conocimiento científico, la razón, hasta las regiones más apartadas del territorio. Junto con tales luces era necesario divulgar, difundir y acendrar la enseñanza de la historia nacional, cuyas referencias de origen estaban siendo escritas casi simultáneamente a su conversión en disciplina escolar. Se trataba pues de generar identidad nacional, de darle cuerpo y alma a una nación llamada México para lo cual era necesario inventarse rasgos y proveniencias comunes. (VÁZQUEZ, 2000; VON MENTZ, 2000).

La estrategia para la creación de una comunidad imaginada que habría de llamarse México fue eficiente. Consolidada a lo largo del siglo XX por los gobiernos postrevolucionarios, hoy en día goza de cabal salud y continúa cumpliendo su función identitaria alrededor de un concepto de mexicanidad cuya historicidad ha sido objeto de estudio específico desde hace más de medio siglo. (Cfr. PAZ, 1978; BARTRA, 1987). Baste un ejemplo: como uno de tantos colofones de los fastuosos festejos a nivel nacional del Bicentenario de la Independencia y Centenario de la Revolución, celebrados en 2010, la Universidad Pedagógica Nacional convocó en el 2011 a un concurso de ensayo entre sus estudiantes con el tema de “¿Qué significa hoy en día ser mexicano?”. Un primer balance de los resultados mostró que en su mayoría los alumnos de esta institución de educación superior (jóvenes del siglo XXI) continúan concibiendo la idea de la mexicanidad con los valores imaginados y construidos en el siglo XIX. La escuela, en la parte 
que de esto le toca, ha sido altamente eficiente. La ausencia de reflexiones sobre las responsabilidades democráticas y ciudadanas como elementos definidores de una noción contemporánea del ser mexicano era evidente, la falta de crítica sobre el propio sentido de la mexicanidad, su ubicación como una construcción histórica con objetivos ideológicos identitarios alrededor de un proyecto político específico de nación no fue motivo de reflexión para los estudiantes de esa institución de educación superior que aceptaron el reto de escribir sobre lo que para ellos hoy en día significa ser mexicano. Los tufos rancios de un patriotismo ramplón permearon muchos de los escritos. No es culpa de los alumnos, ellos simplemente han sido bien educados dentro del sistema escolar que, a pesar de poseer otro tipo de deficiencias, continúa cumpliendo a cabalidad esa tarea ideológica identitaria.

\section{Los docentes}

Sabemos que somos seres históricos, que nuestro sistema de valores debe explicarse sólo y estrictamente en ese mismo sentido. Lo que creemos, pensamos, anhelamos, deseamos, amamos, odiamos, etcétera, debe ser ubicado como expresión de nuestro ser social, del sentido que nuestra participación tiene en una sociedad específicamente histórica en términos cronológicos, geográficos, políticos, culturales, etc.

$\mathrm{Al}$ bosquejar ese marco referencial histórico cultural, podemos entonces visualizar a la función docente tan sólo como una expresión más del ser social que es el maestro(a). Él docente es un individuo partícipe de una sociedad histórica específica que posee un andamiaje simbólico social igualmente concreto, en el que los profesionales de la educación viven, conviven y al que se espera contribuyan a reproducir, dentro del cual su conocimiento histórico (y sobre la historia) es un factor que igualmente han abrevado socialmente. La labor del docente -como la de cualquier otro integrante de la sociedad- constituye una forma de la reproducción simbólica hegemónica, no sólo porque el sistema educativo pueda ser catalogado, según vimos arriba, como uno más de los medios a través de los cuales se crea y recrea ese andamiaje, esos factores político culturales compartidos, sino por el simple hecho de que el individuo docente es parte de la sociedad.

El maestro de historia reproduce y es afín a una cierta forma de valores históricos no porque sea profesional de la educación especializado en una disciplina, sino porque en primerísimo lugar es ciudadano de una sociedad con esos valores. Así las cosas, esperar que ese docente de historia motu proprio realice un ejercicio crítico de fondo sobre qué tipo de historia está enseñando y para qué lo está haciendo es exigirle una responsabilidad ajena a su ser social e incluso 
contradictoria en función de su capacitación profesional. La tensión generada es muy fuerte y tiene su explicación en el doble rol que el maestro encarna, que de facto lo convierte en una especie de bicéfalo cultural o siamés social, ya que en un sólo cuerpo habitan:

a) Un ciudadano común, igual al resto, en el cual se expresan valores, costumbres, estereotipos, sentido común, actitudes, complejos e incluso patologías sociales que conforman la carga cultural históricamente específica de esa sociedad a la que el individuo pertenece, $y$

b) El profesional de la educación al cual se le asigna la responsabilidad social de ser quien transmita y reproduzca en su actividad laboral diaria los mejores valores que la sociedad ha instituido como positivos, justos y necesarios.

Si bien a nivel teórico y desde una perspectiva romántica del quehacer educativo se esperaría que ambos niveles fueran expresiones específicas de un mismo contenido simbólico, la realidad nos muestra que ello no es así. En términos éticos podría plantearse que dentro de ese binomio se evidencia de manera clara la diferencia entre el deber ser (el maestro como figura emblemática de la formación y de la cultura) y el ser real (el ciudadano partícipe de una sociedad definida por la ausencia de una cultura democrática). Vemos pues que, por una parte, se encuentra la actitud que se espera del individuo como docente, esto es, como personal profesional encargado de transmitir esa parte específica del bagaje cultural de la sociedad que es el conocimiento; y por la otra, los sistemas de valores que dicho individuo tiene y reproduce como sujeto social, entre los cuales su idea del significado de ser ciudadano, así como de lo que es la historia, la democracia, sus devenires, protagonistas, etcétera, son parte medular.

En esa complejidad binaria nos hemos debatido por mucho tiempo, pues por lo general existe la pretensión de que el maestro se reconozca a sí mismo y sea reconocido por la sociedad como un ser distinto, como el prototipo del individuo que debiera proveer a sus educandos de cultura, conocimientos, educación, luz, en fin, constituirse en un ejemplo para ellos y para la sociedad toda (sacerdocio laico). Lo romántico de la visión no quita lo imprudente de haber pensado así y, sobre todo, de haber delegado tan grandes responsabilidades de esa manera.

Además, como si eso fuera poco, hay un punto que por sabido parece olvidarse cuando desde el ámbito de las mejores intenciones se plantea la ineludible responsabilidad que la escuela en particular, y todo el sistema educativo en general, tiene de procurar y generar la constitución de individuos (jóvenes y niños/as) con "espíritu cívico", buenos ciudadanos conocedores y practicantes de sus derechos, críticos, participativos en la toma de decisiones y demás actitudes de vida en las que se concretaría la construcción de competencias para la convivencia democrática. Bajo el entusiasmo por planear, diseñar y llevar a 
cabo tan profunda transformación educativa cultural, se hace caso omiso de los ya añejos análisis que evidencian la manera en la que la escuela fue construida para reproducir ciertas formas específicas de dominio. Recordemos que históricamente la escuela (todo el dispositivo de su funcionamiento) está pensada exactamente para lo contrario a la construcción de una práctica democrática; desde los espacios arquitectónicos hasta las formas de concebir los procesos de enseñanza aprendizaje; desde los contenidos curriculares hasta los sistemas de poder, toma de decisiones y la gestión y resolución de problemas.

De la misma manera en que es necesario tener presente el rol que la institución educativa juega en la reproducción de una hegemonía política cultural específica, también es urgente y fundamental desacralizar la imagen del maestro y reivindicar su humanidad, entendiendo por ella lo que hemos estado desarrollando hasta aquí: el maestro es un ser social partícipe de una cultura y, como condición para poder ubicar los alcances y límites de su accionar profesional, debe ser capaz de verse a sí mismo y a su práctica docente en esa definición, dentro de esos márgenes y posibilidades. Si la misoginia, el racismo, el apoliticismo o el maniqueísmo político, la corrupción, el autoritarismo, es lo que impera en la sociedad, es evidente que los trabajadores de la educación (al igual que los demás) no podrán abstraerse de vivir bajo esa carga cultural, por lo cual para ellos y para todos es un enorme y difícil reto asumir una realidad y actitudes distintas.

Sin duda el contrasentido más grotesco en donde se ejemplifica lo anterior, que de tan evidente deviene invisible, es el constituido por la trágica ironía política y ética encarnada en el hecho de que en México institucionalmente se pretenda que los mismos sujetos que laboralmente han sido incapaces de darse formas sindicales democráticas sean los responsables de construir en la infancia y juventud de nuestro país las competencias necesarias para la convivencia democrática a fin de formar a los nuevos ciudadanos. Si bien lo anterior corresponde a los maestros responsables de la materia de formación ciudadana, el caso de los responsables de impartir los programas de historia también es partícipe de estas contradictorias características, en la medida en que la historia que se enseña tiene como objetivo fundamental la creación de una identidad nacional vinculada a una noción específica de ciudadanía. Así, en la escuela los contenidos de historia nacional contemplados en los programas son reforzados por la existencia de innumerables rituales cívicos, conmemoraciones de efemérides, etcétera. Los maestros han sido los responsables de hacer patria, de constituir nación. A ellos se les asigna esa enorme responsabilidad que habrán de desarrollar desde su trinchera, para lo cual se les arma en lo particular con la historia patria y, en lo general, con todo el aparato escolar, constituyéndose así un claro ejemplo de dialéctica social: la escuela los forjó profesionalmente 
como actores protagonistas en la construcción de una identidad patria y, a su vez, ellos coadyuvan a consolidar la idea de que la escuela no solo es un "templo del saber" sino en primerísimo lugar un espacio conformador de esa misma identidad nacional.

Las ceremonias cívicas al iniciar los lunes las labores escolares (uso de uniforme de color blanco, honores a la bandera, interpretación del himno nacional, marchar hacia los salones mientras se escuchan música típica o marchas militares, etcétera.), las conmemoraciones de múltiples efemérides en donde toda la escuela se ve involucrada, van de la mano de la historia que se estudia y de la forma de enseñarla. Los maestros, repito, más allá de ser profesionales de la educación a los que se les ha asignado esa responsabilidad, en primer lugar fueron hombres y mujeres que como alumnos pasaron por esa institución, en la cual crecieron, y conformaron su noción de ciudadanía bajo tales características. Así, comprendemos mejor la manera en que para ellos (y para toda la sociedad) es muy difícil tomar distancia de tal referencia acerca de la utilidad de la historia como disciplina escolar.

Las fuertes polémicas que se generan cada vez que se habla de la necesidad de ajustar los planes y programas de estudio de historia dentro del sistema escolar, tienen su explicación en la profunda convicción de que la historia sirve fundamentalmente para inocular un sentido identitario patrio en los alumnos. De hecho algunos especialistas llegan a hablar de "guerras culturales sobre la enseñanza de la historia" (CARRETERO, 2007, p. 159) pues, en efecto, lo son. El peso de dichos valores hegemónicos es tan importante que incluso historiadores profesionales hoy en día continúan tomando partido en ese tenor. Un ejemplo:

Agresivo o defensivo, el nacionalismo presupone la afirmación de lo propio a costa de lo ajeno. Es una actitud que pertenece a la esfera del poder. El patriotismo, en cambio, es un sentimiento de filiación: pertenece a la esfera del amor. Pero una vez pasado el umbral de la infancia, plantada la semilla del amor por este país, debe sobrevenir un sano y paulatino desencanto sobre la naturaleza de los "héroes". La duda metódica y la búsqueda de la verdad deben desplazar a la admiración sentimental. (KRAUZE, 2010, p. 20-21).

De acuerdo a Enrique Krauze la historia tradicional que se enseña en la escuela es válida para imbuir en los niños el amor a la patria aunque ya después, según esos infantes vayan creciendo, deberá ser sustituida por otras versiones más plurales y diversas. En el proceso de concebir la historia que debe ser enseñada en el sistema de educación básica, este autor, reconocido difusor del conocimiento histórico, se muestra incapaz de imaginar una historia que se salga de los cánones institucionales y de las utilidades asignadas a la historia institucionalizada. La posibilidad de replantear todo, absolutamente todo el sistema escolar y, por 
ende, la concepción de la historia que ahí se enseña, está fuera de su partitura crítica, ausente en su horizonte de visibilidad analítico. A los niños, nos dice, hay que darles lo que se merecen por su condición infantil: historias lineales, unívocas, básicas, patriotas, falsas. La idea de que en la escuela y desde las edades más tempranas se empiece a identificar y a aprovechar (pedagógica, cultural y políticamente) la existencia de la diversidad para pensar nuevas competencias que el conocimiento histórico habría de fomentar vinculadas a nociones de ciudadanía, comprensión de los problemas del presente, etcétera, queda fuera de su ámbito de observación.

La enseñanza de la historia cumple su objetivo social. Los maestros de historia, de formación cívica y ética, y la escuela en general, reproducen los valores hegemónicos respecto a la utilidad y funciones de la enseñanza de la historia. No tendría por qué ser de otra forma, la hegemonía funciona, es eficiente.

\section{La concepción de la historia}

La historia es una disciplina cuya enseñanza dentro del sistema escolar surgió con la necesidad de construir valores identitarios alrededor de los nacientes Estados nación. La escritura de las historias patrias, junto con su difusión a través de los sistemas educativos, se explica a partir de la necesidad histórica de los nacientes Estados nación de generar sentidos de identidad entre una población caracterizada fundamentalmente por su diversidad. La conocida sentencia de los nacionalistas italianos expresada claramente por Mazzini al expresar: "Hemos creado Italia, ahora tenemos que crear italianos", refiriéndose a la necesidad de construir y consolidar una "conciencia italiana" que imbuyera una identidad nueva y particular en los sujetos que habrían de ser considerados a partir de ese momento como ciudadanos del nuevo estado, puede ser utilizada para comprender en general la escritura de las historias patrias así como el rol central que estas habrían de jugar en la curricula de los sistemas escolares nacionales.

Junto con la historia nacional, la imposición como disciplina escolar de una historia denominada "universal" o "mundial" también cumple un rol identitario específico aunque de índole más general. Este sería la vinculación ideológica con un y sólo un sentido de devenir, referido a valores netamente occidentales plasmado en concepciones específicas de tiempo lineal, expresiones de un sentido de modernidad para el cual el pasado, el presente y el futuro se explican en función del anhelo de progreso, noción entendida como la capacidad de desarrollo económico de características fundamentalmente capitalistas.

Sin embargo, hoy en día junto con la crisis de los universales entre los cuales se cuenta la modernidad y la idea de Nación, se han puestos grandes signos de interrogación frente a las comprensión tradicional de las funciones 
sociales de la historia como disciplina escolar. La idea de que el conocimiento histórico en particular, y social en general, debiera estar perfilado hacia la construcción de competencias para la convivencia ciudadana hace que los puntos referenciales de la enseñanza de la historia se diluyan frente a la necesidad de incluir a las diversidades en el horizonte de las responsabilidades ciudadanas en la construcción de una sociedad democrática moderna.

La ciudadanía se encuentra en la valoración de la pluralidad, el uso del tiempo libre, el respeto y cuidado del legado heredado del pasado y la formación de una conciencia histórica. A estas competencias ciudadanas se les agregan las competencias democráticas que no son específicas de la historia o de las ciencia sociales, sino genéricas. Las competencias destituyen a la historia nacional del lugar privilegiado que ocupaba en la formación del ciudadano a través de la identidad nacional, pero no rompe de lleno con el código disciplinar sedimentado que patrimonializa el pasado. (PLÁ, En prensa, p. 71).

Hablamos entonces de la necesidad contemporánea de recuperar la diversidad, de reconocer la pluralidad. La historia que hoy se enseña en la escuela mexicana, más allá de las buenas intenciones expresadas en las presentaciones de los planes y programas de estudio respectivos, sigue siendo la misma de siempre, continúa padeciendo de los límites que la definieron desde el siglo XIX, ello a pesar de la crítica a la que ha sido sometida desde hace lustros. Veamos la manera en que la univocidad de la historia elimina la diversidad, los cual en los hechos significa la renuncia de la propia historia al axioma que ella postula para el análisis de los hechos sociales: la historización.

¿Por qué en pleno nuevo milenio los habitantes de América continuamos refiriéndonos a las regiones del continente asiático como el "lejano oriente", si basta ver cualquier globo terráqueo o planisferio para percatamos de que tales regiones quedan del otro lado del océano Pacífico, esto es, en nuestro oeste? ¿Cuál es la razón de que después de quinientos años seguimos dando por cierto y, por tanto, utilizamos en nuestra cartografía básica un planisferio que tergiversa de manera grotesca y grosera las dimensiones reales tanto de los países, como de los hemisferios y las regiones? ¿Por qué, además de los ineludibles cursos de historia nacional, se imparten cursos de una historia universal que en el mejor de los casos se refieren única y exclusivamente a una historia de ciertas civilizaciones lo cual en rigor ni siquiera permite que sean catalogada bajo el concepto general de "europea"? ¿cómo explicamos el hecho de que a pesar de que México se caracteriza por la existencia de una multiplicidad de culturas, la historia nacional que se aprende dentro del sistema educativo hace caso omiso de ellas a pesar de las "buenas intenciones" programáticas de reconocer dicha diversidad? ¿Por qué en México idolatramos a los indios muertos, habitantes de un lejano pasado prehispánico, pero defenestramos a los indios vivos de nuestro presente 
globalizado? ¿Cuál es el papel (si existe) de los otros en la historia, así en general, que aprendimos y enseñamos?

Las preguntas son una infinidad. La historia, esa imagen que una sociedad construye de sí misma y su devenir, debe empezar a verse en el espejo de sus propios límites históricos, sólo así será posible identificar la necesidad de avanzar hacia la construcción de nuevas competencias educativas en lo referido al aprendizaje de lo que tradicionalmente se conocen como ciencia sociales. La crítica, el ejercicio intelectual por antonomasia, debe en primerísimo lugar dirigirse hacia las nociones que de tan arraigadas han devenido invisibles.

Los axiomas del estilo "la historia la escriben los vencedores" a fuerza de ser repetidos han sido vaciados de contenido convirtiéndose en lugares comunes, áridos y deshabitados, abundantes en el páramo intelectual de nuestra contemporaneidad. Se naturalizan frases hechas que terminan por invisibilizar lo que debiera ser evidente: su historicidad. En efecto, la historia la escriben los vencedores, pero ello no quiere decir que no existan otras historias que están ahí, y siempre lo han estado, como ríos subterráneos cuyo caudal súbitamente brota en manantiales que alimentan nuestra sed de identidad. Estos torrentes culturales profundos se entrecruzan en los cimientos de la sociedad contemporánea y terminan por erosionar los anhelos de uniformidad que la modernidad ha impuesto. Una vez más su corriente se convierte en elemento vital para la vida que, por definición, es diversa y multiforme.

Una noción contemporánea de ciudadanía implica necesariamente el reconocimiento de esa diversidad. La educación, la enseñanza de la historia, habrían de empezar por poner en el centro de su atención la posibilidad de la alteridad empezando por la construcción de las narrativas históricas, lo cual implicaría comenzar a formar en los sujetos el reconocimiento de la otredad como elemento definidor de su propia mismidad:

Pensar la formación de sujetos ciudadanos o sea, la constitución de sujetos políticos, de sujetos morales, es pensar la identidad en la relación con los otros. Para ello habrá que dejar atrás la idea del ciudadano como mero sujeto prudente y autónomo para entenderlo desde una posición de alteridad, como negociador de sentidos, y por ello como un esfuerzo de construcción con otros en el encuentro con el mundo. La formación de una ciudadanía resignificada. (FERNÁNDEZ, 2007, p. 27).

Es falso que recientemente hayamos empezado a vivir juntos pues siempre lo hemos hecho. Más bien muchas certidumbres monoformes se han resquebrajado -cuando no derruido completamente- a causa de la irrupción de los otros que durante un periodo históricamente definido y explicable se pretendió hacer desaparecer bajo multitud de caretas de toda índole, desde la erradicación 
violenta hasta la integración política, económica y/o cultural. Los imperios, los mestizajes, la supresión política artificial de las nacionalidades, la represión violenta y simbólica sobre los diferentes, son algunas de esas maneras mediante las cuales se pretendió eliminar / integrar la idea de la existencia de lo otro en aras de consolidar una noción unívoca del devenir de la humanidad. Una sola voz dictó la historia, una sola mirada se impuso sobre el pasado, su reconstrucción y su enseñanza. Una exclusiva manera de ver al mundo y su estudio y posible comprensión. Al momento en que reaparece lo que aparentemente se había desvanecido, la otredad encarna aquí y ahora obligándonos a pensar todo de nuevo.

No es fácil vernos desde esa nueva perspectiva. Los discursos, las formas hegemónicas se han arraigado lo suficiente para ser eficientes, lo cual implica imbuirse de naturalidad y vestirse con los ropajes de la universalidad. La hegemonía devenida en conformación cultural genera nociones profundas difíciles de desenraizar. Un ejemplo son las fronteras nacionales. Los niños mexicanos aprenden en la escuela que en el norte la frontera es el Río Bravo y en el sur el Suchiate; dentro de esos parámetros geográficos se ubica la posibilidad de ser mexicano, bajo esa aura se elimina la diversidad de los grupos étnicos cuyos asentamientos no reconocen tales líneas divisorias. La historicidad del Estado nación se diluye detrás de una pretendida figura universal: la nación, la patria. La artificialidad de las fronteras políticas se erige de manera universal por encima de las historicidades específicas de los grupos humanos.

La pretensión señalada en los programas educativos de educación básica y media de rescatar esas historias específicas no pasa de ser simple un enunciado demagógico políticamente correcto que intenta cubrir el expediente de hacerse eco de las corrientes de moda sobre la interculturalidad, el multiculturalismo, la diversidad, etc. Todos nosotros lo sabemos: los programas de historia continúan fortaleciendo una sola visión del mundo, imbuyendo ciertas y específicas nociones sobre patria y universalidad. Cualquiera que confronte los objetivos expresados en la presentación de dichos programas académicos contra los contenidos específicos que deben ser estudiados y aprendidos por los niños y jóvenes mexicanos, se percatará inmediatamente de la aguda esquizofrenia conceptual que los caracteriza.

La historia que se enseña a lo largo del sistema educativo mexicano sigue manejándose dentro de tales axiomas. La historia es un mecanismo más de construcción de cierta imagen del mundo, de hegemonía política. Paradojas y dobles raseros de la racionalidad crítica. La ciencia (histórica) que debiera ser crítica por definición, cede la plaza frente al poder. Luego entonces la historia como disciplina (y su enseñanza) requiere ser historizada. Una educación para la diversidad asumiendo que este concepto es axial para la construcción 
de una ciudadanía moderna vinculada a nociones democráticas en sentido amplio, no será posible mientras la historia se siga concibiendo sólo bajo una forma epistemológica y discursiva, esto es, como una de las hijas entenadas de la racionalidad que garantiza la única manera de lograr el conocimiento real, objetivo, verdadero, etc. del pasado. La manera en que estos discursos devinieron hegemónicos y se imbricaron a funciones políticas como la construcción de identidades nacionales y occidentales, puede y debe ser explicada históricamente, como requisito ineludible para poder plantear la necesidad de transformar los usos asignados a la historia, de tal forma que se abra la puerta para poder concebir a la enseñanza de las ciencias sociales en educación básica desde nuevas perspectivas referidas a la construcción de competencias para la convivencia y la participación democrática de los individuos.

La diversidad no debe quedarse en el nivel discursivo o remitirse al ámbito exclusivamente político cuando se habla de cultura, sino que, siendo consecuentes, ha de reconocer, respetar, valorar y enriquecerse a partir del establecimiento de relaciones multidireccionales con otras formas de concebir el pasado que no tienen nada que ver con nociones perfectamente historizables como el tiempo lineal (pasado-presente-futuro), nación, patria, progreso, democracia y demás conceptos característicos de una concepción de historia vinculada al encumbramiento de la ciencia como el discurso hegemónico.

El desdén y ninguneo sobre otras formas de concebir al mundo, y por tanto, devincularse con él en lo que desde la modernidad occidental llamamos epistemología, significa una forma específica de racismo, en la medida en que desde un valor universal (la ciencia y sus encarnaciones positivistas en lo que concierne a las humanidades), discriminamos (calificamos de inferior) cualquier otro tipo de conocimiento, de vínculo con la naturaleza y, en suma, de concepción del mundo.

Los conceptos nodales dentro de la manera tradicional y vigente de entender a la historia y las formas de abordarla metodológicamente son expresiones claras de esa universalización de lo particular. Nociones como universal, occidental, moderno, nación, patria, tiempo, ley, causalidad, verdad, orden y, por supuesto, historia (amén de muchísimos más) son propuestos para leerse de manera unilineal. Al haberse impuesto desde una situación de poder el discurso triunfador culturalmente es asumido acríticamente: lo particular se convierte en universal y la historia concebida como ciencia renuncia a sus propios postulados básicos entre los cuales el primero es la historización de todo, empezando en primerísimo lugar por ella misma.

Vemos pues que es necesario hacer la historización de la historia, es decir, ubicar en términos culturales, sociales, políticos, filosóficos, económicos, etc., el 
surgimiento, consolidación y reproducción de una forma de concebir lo que es el mundo, la sociedad y, por tanto, su estudio tanto en su presente, más cuanto de su pasado. Sólo así podremos concebir a la historia desde una perspectiva que recupere a la diversidad y esté pensada para la diversidad, cuestión fundamental para la construcción de los cimientos de una ciudadanía moderna.

\section{¿Qué hacer?}

¿Educación o formación para la ciudadanía? ¿Qué concepción de democracia está detrás de las diferentes acepciones de ciudadanía? ¿Cuál es el significado de ser ciudadano, cómo se ha modificado históricamente, a qué ámbitos analíticos él hace referencia? ¿Cuáles son los agentes responsables de formar para la democracia? ¿Existen esos agentes? Una multitud de preguntas que hacen evidente que el tema elegido es un texto abierto que está siendo escrito y categorizado día a día. Algunas de las múltiples determinaciones de este objeto de estudio, sugerirían amplias líneas reflexivas:

- Desde la perspectiva filosófica del significado ético y moral de la constitución del ciudadano, hasta el análisis puntual de cómo la historia que se enseña en el sistema educativo nacional reproduce y fortalece una noción específica de ciudadanía.

- De la reflexión sobre la manera en que la educación juega o no un rol particular (y sus límites) en la formación de ciertos valores democráticos, hasta el desglose minucioso de las políticas educativas que pretenden impulsar la formación de valores y actitudes democráticas propuestas para Latinoamérica por diversos organismos internacionales.

- Del análisis de las contradicciones culturales que el tema contiene a la crítica específica de la escuela y sus agentes como formadores específicos de valores no democráticos.

Vemos entonces que el tema rebasa los estrechos ejes a los que por lo general se le pretende reducir. La práctica democrática no se restringe a la enseñanza aprendizaje en términos teóricos de una serie de actitudes y competencias para la convivencia-, tampoco a la participación periódica en una actividad específica para elegir todo lo que sea elegible (la trillada idea la participación en la "fiesta cívica"). No, la democracia es una práctica que, al convertirse en costumbre, se volvería manera de ser. Así, tanto la escuela en su labor de educación para la democracia, como los procesos electorales concebidos como la actividad a la que comúnmente se refiere y reduce la obligación ciudadana de participación política democrática, muestran toda su limitaciones históricas, culturales y, en una palabra, políticas. Por ello es que el desarrollo específico de una perspectiva 
crítica, general y profunda sobre la institución escolar, ayuda a concebir de manera amplia el espacio de responsabilidad donde debemos ubicar el tema de la formación para la ciudadanía.

Puntos ineludibles para la superación del conflicto entre la existencia de valores hegemónicos que contradicen los elementos básicos de convivencia moderna (tolerancia, respeto a la diversidad, igualdad de género, pluralismo, respeto y ejercicio de los derechos humanos y ciudadanos, etcétera) que deben ser construidos por el sistema educativo, son:

Primero: la identificación y el reconocimiento de los valores hegemónicos y su historicidad (hacer visible lo invisible);

Segundo: la identificación de la forma en que en nuestra cotidianidad y por lo menos en los niveles personal, familiar, profesional y ciudadano reproducimos dichos valores.

Tercero: asumir la existencia de la contradicción entre lo que decimos / postulamos / enseñamos y lo que hacemos (personal y profesionalmente), como requisito indispensable para poder empezar a resolverla en ese primer plano, en esa esfera de la individualidad particular y única (yo).

Lo anterior, en resumen, implica modificar las prácticas de enseñanza y aprendizaje poniendo en el centro ya no la transmisión de valores que siendo históricos devienen (se presentan y asumen como) universales (nación, mexicanidad, democracia, progreso, etc.), sino en la posibilidad de problematizar lo que somos a partir del reconocimiento de nuestros límites y especificidades históricas tanto a nivel social (nosotros, el sujeto particular como uno más del grupo social) como individuales (yo), lo cual sólo es posible si se reconoce la relatividad de tales valores, lo que a su vez nos permitirá concebir y aceptar la posibilidad del cambio.

Reconociendo la importancia de la individualidad, de lo particular más singular, comprenderemos la enorme posibilidad libertaria que, entre otras cosas, nos ha dado la noción de diversidad redescubierta en los últimos lustros. Hacernos conscientes de que en lo pequeño puede estar (y está) lo grande es poner de cabeza las expectativas políticas de transformación social en las que la mayoría de nosotros crecimos intelectualmente. Asumir esa frontera primera que es nuestro propio yo nos obliga a modelarnos, a definirnos, a distinguirnos, en suma a crear nuestra propia identidad, es decir, asumir en todas sus consecuencias esa "soberanía del individuo sobre sí mismo, cuya abdicación es imposible". (VÍCTOR HUGO, 1980, p. 784.) En esto radica la clave hoy en día del significado de "ser ciudadano". Así, a manera de círculos concéntricos, esta reflexión y ejercicio crítico se va extendiendo poco a poco hacia los diversos espacios sociales en los que nos movemos (familia, escuela, barrio, comunidad, 
etc.) abriendo así la posibilidad real de que el discurso sobre la ciudadanía, es decir, sobre los derechos y obligaciones que el individuo tiene en su sociedad, la manera de resolver conflictos, el ejercicio del poder y la autoridad, etc. empiece a dejar de ser simple letra descriptiva para iniciar su conversión en práctica cotidiana.

Hoy es necesario edificar desde las bases mismas, desde sus aspectos más elementales, un ejercicio, una práctica diaria democrática. No faltará quien vea en esta reflexión algo así como una recuperación romántica y trasnochada de alguna utopía supuestamente extinguida. Pero el que una comunidad de una de las regiones más olvidadas por la modernidad, haya planteado en 1994 como prioritaria la necesidad educativa de que sus hijos fueran educados de manera integrada -

[... dando una disciplina respetuosa a los derechos del niño, en la que sin maltrato, niños y niñas aprendan a respetar a los otros, sin golpearlos ni humillarlos; aprendan a reconocer sus errores y pedir disculpas, aprendan a respetar la naturaleza [...] aprendan palabras dulces, buenas y respetuosas de los otros, y no palabras que dañen la dignidad de los demás, [...] aprendan a respetar las costumbres de los otros: su forma de vestir, de hablar, de pensar, de creencias y gustos, reconociendo el derecho a ser diferentes, [...] aprendan a compartir sus experiencias y objetos, aprendan que los problemas se resuelven en el diálogo [...], aprendan a participar y a hacer propio el proyecto educativo [...] (CONVENIO EDUCATIVO, 1994, s/p).

- constituye la forma idónea de asumir que tolerancia, la pluralidad, la responsabilidad, la ciudadanía, etcétera, no son conceptos abstractos; que la otredad (su reconocimiento y aceptación como elemento definidor del nosotros) adquiere un sentido concreto, específico, para la democracia. En efecto, es posible otra hegemonía cuyos objetivos no sean la despolitización, la homogenización, la intolerancia o la desmovilización consumista y hedonista característica del mundo de la globalización.

La formación ciudadana, la creación de competencias para la convivencia democrática es, en efecto, una cuestión de aprendizaje que tiene que ver con su ejercicio diario y cotidiano, que no se remite exclusivamente a lo escrito en los libros, a los contenidos señalados en los programas escolares de historia y formación cívica y ética, sino más bien es un aprendizaje diario (práctica cotidiana) que si bien pasa por el espacio escolar lo rebasa y se extiende hacia todo el espectro de la vida social. La utilidad contemporánea, moderna, de la enseñanza de la historia y de la formación cívica debe ser visualizada bajo este lente, desde esta convicción filosófica, política y social. 


\section{Colofón}

Curiosamente le debemos a un poeta una de las definiciones más claras del significado que la democracia habría de tener en las sociedades contemporáneas:

Ahora se habla mucho de la democracia en México sólo que, en general, se le reduce a una serie de ideas y conceptos. No, la democracia es también una práctica. A su vez, las prácticas sociales, al arraigarse, se convierten en hábitos y costumbres, en maneras de ser. Para que la democracia funcione realmente debe haber sido perfectamente asimilada e incorporada a nuestro ser más íntimo. La democracia debe transformarse en una vivencia. Esto es lo que, todavía, no sucede en México. (PAZ, 2003, p. 572).

La democracia no es la panacea, es únicamente una forma de convivencia, de resolución de conflictos en donde algunos ganan y otros pierden, en la cual no siempre se obtiene el resultado anhelado pero en la que las reglas de convivencia existen, son claras y se respetan. Ser ciudadano, entre otras cosas, significa aprender a vivir con la frustración y el gozo que dichas prácticas suponen. Ello significa un trastocamiento radical tanto de las concepciones sobre la escuela y la formación, como de las prácticas político culturales democráticas de las cuales, por cierto, la institución educativa no puede (no debe) escapar, pero de las que tampoco ella es la única responsable de fomentarlas y desarrollarlas. Dentro de ella la historia y su enseñanza (junto a la educación cívica) habrá de reajustar la concepción de su utilidad como generadora de ciertas identidades referidas a lo nacional y lo occidental, abriendo paso a una visión mucho más compleja (y moderna) de su función social, la referida a la construcción de las competencias para la ubicación del sujeto como un individuo consciente de sus derechos y obligaciones para con la sociedad a la que pertenece, los cuales habrá de ejercer y cumplir en beneficio de los otros, lo que significa abonar en su propio beneficio como ciudadano.

\section{Referencias}

BARTRA, R. La jaula de la melancolía. México: Grijalbo, 1987.

BERNAL, M. Atenea negra: las raíces afroasiáticas de la civilización clásica. Barcelona: Crítica, 1993.

CARRETERO, M. Documentos de identidad: la construcción de la memoria histórica en un mundo global. España: Paidós, 2007.

CONAFE. Consejo Nacional de Fomento Educativo. Educación intercultural: una propuesta para población infantil migrante. México: CONAFE, 1999.

CONDE, S. L. Educación ciudadana: de la política educativa a la formación política.

Cuadernos México, n. 1, p. 67-77, 2009. 
CONVENIO EDUCATIVO que se celebra entre las madres, los padres y el Comité de Educación Autónoma de la Comunidad de Monte Flor, Municipio Autónomo Tierra y Libertad.Chiapas: México, y el Instituto de Investigaciones Pedagógicas, A.C. 1994.

CUESTA, R. Historia, educación y didáctica crítica. Consideraciones fedicarianas. Disponible en: <http://www.nebraskaria.es/Nebraskaria/Sobre_Fedicaria_files/Burgos. pdf>. Acceso en: 10 jun. 2013.

DE CERTEAU, M. La escritura de la historia. México: Universidad Iberoamericana, 1993.

DE CERTEAU, M. Historia y psicoanálisis. México: Universidad Iberoamericana / Instituto Tecnológico de Estudios Superiores de Occidente, 1995.

FERNÁNDEZ, A. La formación de una ciudadanía resignificada. In: VIDALES, I.; MAGGI, R. E. (Comps.). La democracia en la escuela: un sueño posible. México: CECyTE / NLCAEIP, 2007. p. 27-37.

FERRO, M. Cómo se cuenta la historia a los niños del mundo entero. México: Fondo de Cultura Económica, 2000.

FLORESCANO, E. La función social de la historia, México: Fondo de Cultura Económica, 2012.

FREYRE, P. La educación como práctica de la libertad. México: Siglo XXI, 1971.

KRAUZE, E. De héroes y mitos. México: Tusquets, 2010.

LOEWEN, J. Lies my teacher told me. USA: The New Press, 2007.

MAALOUF, A. Identidades asesinas. España: Alianza, 2008.

PAZ, O. El laberinto de la soledad. México: Fondo de Cultura Económica, 1978.

PAZ, O. Itinerario. México: Fondo de Cultura Económica, 1993.

PAZ, O. Tela de juicios: obras completas. Tomo 15. México: Fondo de Cultura Económica, 2003.

PLÁ. S. Nuevas y viejas narraciones en la enseñanza de la historia: los casos de México y España. Cuadernos México, n. 1, p. 19-28, 2009.

PLÁ, S. Ciudadanía y competitividad en la enseñanza de la historia: los casos de México, Argentina y Uruguay. México: Universidad Iberoamericana. En prensa.

RODRÍGUEZ, X. Una historia desde y para la interculturalidad. México: Universidad Pedagógica Nacional, 2008.

RODRÍGUEZ, X. Historia y diversidad. El lugar de la otredad en la reconversión del paradigma imperante.Cuadernos México, n. 1, p. 29-38, 2009.

SAID, E. Orientalismo. Barcelona: DeBolsillo, 2004a.

SAID, E. Cultura e imperio. España: Anagrama, 2004b.

TENORIO, M. Historia y celebración. México: Tusquets, 2009.

VÁZQUEZ, J. Nacionalismo y educación. México: El Colegio de México, 2000.

víCTOR HUGO. Los miserables. México: Porrúa. Colec. “Sepan cuantos”, 1980.

VON M. B. Nación, Estado e identidad. Reflexión sobre las bases sociales del estado nación 
en el México del siglo XIX. In: VON M. B. (Coord.). Identidades, estado nacional y globalidad. México: CIESAS, 2000. p. 33-93.

WHITE, H. Metahistoria: la imaginación histórica en la Europa del siglo XIX. México: Fondo de Cultura Económica, 1992a.

WHITE, H. El contenido de la forma: narrativa, discurso y representación histórica. España: Paidós, 1992b.

Recebido em 25/02/2013

Versão final recebida em 13/06/2013

Aceito em 17/06/2013 